\title{
Der Leuchtturm von Pharos - ein spätes Weltwunder
}

\author{
Korana Deppmeyer
}

Während die ältesten schriftlichen Zeugnisse zu den Sieben Antiken Weltwundern bereits in das 2. vorchristliche Jahrhundert datieren ${ }^{1}$ und zahlreiche folgende Wunderlisten ${ }^{2}$ über einen langen Zeitraum ein buntes Sammelsurium von Anlagen und Bauten ${ }^{3}$ vereinen, bleibt der Leuchtturm von Alexandria - der Pharos unerwähnt und wird es für nahezu 800 Jahre seiner Existenz bleiben ${ }^{4}$.

Für die Errichtungszeit des Pharos können Vermerke in der Suda ${ }^{5}$ und bei Eusebius $^{6}$ als zeitliche Markierungen gelten, denn dort ist das Jahr 297 v. Chr. bzw. 283/2 v. Chr. genannt, womit der Baubeginn unter dem Lagidenkönig Ptolemaios I. Soter und die Vollendung unter seinem Nachfolger Ptolemaios II. Philadelphos, also eine Bauzeit von 15 Jahren, anzunehmen ist ${ }^{7}$.

Strabon $^{8}$ und Lukian ${ }^{9}$ erwähnen jeweils, dass zur Rettung der Seefahrer der Pharos mit „hohen und hervorleuchtenden Zeichen“ errichtet wurde. Plinius ${ }^{10}$ beziffert die Kosten des Bauwerkes auf 800 Talente $^{11}$ und spricht ebenfalls von den Feuersignalen. Flavius Josephus weiß mitzuteilen, dass der griechische Mathematiker Archimedes $^{12}$ einen Brennspiegel konstruierte, der das Turmlicht am Tag bis zu 300 Stadien $^{13}$ weit auf das Meer warf ${ }^{14}$. Es ergibt sich somit nach Berechnungen für das

\footnotetext{
${ }^{1}$ Das älteste bekannte Schriftstück ist ein fragmentarischer Papyrus, Laterculi Alexandrini. Er benennt die Pyramiden, den Artemistempel von Ephesos und das Mausoleum von Halikarnass. Ob auch der Pharos erwähnt wurde, ist wegen des schlechten Zustandes nicht zu beurteilen. Die erste komplett erhaltene Liste stellt eine mittelalterliche Handschrift dar, die sog. Anthologia Palatina. Ein Abschnitt (IX 58) wird Antipatros von Sidon zugeschrieben, der den Artemistempel von Ephesos, die Mauern von Babylon, den Zeus von Olympia, die Hängenden Gärten der Semiramis, den Koloss von Rhodos, die Pyramiden sowie das Mausoleum von Halikarnass aufführt. Diese Reihe entspricht den späteren ,klassischen' Wunderlisten und zeigt eine beginnende Kanonisierung der Listen. Vgl. zu den Schriftzeugnissen der Weltwunder: Dawid (1968) 66-68; Brodersen (1992); Weltwunder (2003) 9-14.

${ }^{2}$ Die frühen Listen wurden wohl zunächst im Osten - im Seleukidenreich - gefertigt, was die Überzahl der sich einst dort befindlichen Monumente belegt: So werden sowohl die Hängenden Gärten als auch das Stadttor von Babylon in einer Liste genannt. Aus späterer Überlieferung lässt sich auf eine weitere alexandrinische Liste schließen, da nunmehr sowohl die Pyramiden und Theben, die Pyramiden und die Memnonkolosse, oder aber der Pharos und die Pyramiden Erwähnung finden. Vgl. hierzu die zusammengestellten Listen bei Dawid (1968) 66-68; Brodersen (1992) 75-157.

${ }^{3}$ So z. B. der Palast des Perserkönigs Kyros von Ekbatana, der Hörneraltar von Delos oder das Amphitheater in Rom.

${ }^{4}$ Die wohl älteste bekannte Nachricht vom Pharos stellt ein Epigramm des Poseidippos von Pella (um 270 v.) dar. S. auch Fn. 56. Ein weiterer Papyrus, Firmin-Didot (um 161 v. Chr.), beschreibt die Lage des Turmes und seine Beleuchtung als Wegweiser für die Schiffe. Abgedruckt bei Oehler (1920) 163.

${ }^{5}$ Suda s. v. Фó́@oৎ Nr. 114.

${ }^{6}$ Eus. Chron. Can. Arm. Ol. 124 (abgedruckt in: Schoene (1866)).

${ }^{7}$ Die Nachricht von Amm. 22, 16, 9, der als einziger den Bau Kleopatra zuschreibt, ist fehlerhaft.

${ }^{8}$ Strab. 17, 6, 791 .

${ }^{9}$ Lukian. 62.

${ }^{10}$ Plin. nat. 36, 83. Brodersen (1993) 208 spricht hier von der ersten Weltwunderliste, die den Turm erwähnt. Es handelt sich jedoch vielmehr um eine Aufzählung bekannter Bauten und ihrer Besonderheiten.

${ }^{11}$ Diese Information wird auch im 7. Jh. n. Chr. vom Bischof von Sevilla, Isidorus, Orlginum seu Etymologiarum libri, XV, 2, 37 erwähnt. 800 Talente entsprechen ca. 20800 kg Silber: Grimm (1998) 43.

${ }^{12} \mathrm{Zu}$ Archimedes: Schneider (1979); Russo (2005) 216-222.

${ }^{13}$ Das sind ca. 54 km: Clayton - Price (1991) 145.

${ }^{14}$ Ios. bel. Iud. 4, 10, 5.
} 
Bauwerk eine Höhe zwischen 120 und 140 m, was fast der Höhe der Cheopspyramide entspricht, die schon Herodot als architektonische Meisterleistung pries ${ }^{15}$.

Diese Quellen vermitteln den Eindruck, dass vorrangig technische Aspekte wie die Reichweite des Feuers, der Spiegel und die Turmhöhe offenbar als nennenswert, nicht aber so bewunderungswürdig galten, dass sie dem Pharos eine Aufnahme in die Reihe der antiken Weltwunder eintrugen. Dies wird auch insofern offensichtlich, als Strabon längere Zeit in Alexandria lebte ${ }^{16}$ und somit den Leuchtturm kannte, diesen aber keinesfalls enthusiastisch rühmte.

Antike Berichte, die die Gestalt des Bauwerkes wiedergeben, sind nicht überliefert, obwohl der auf einem Felsriff vor der Spitze der Insel Pharos über einer Zisterne errichtete Leuchtturm ${ }^{17}$, der namengebend für eine ganze Gattung werden sollte, als „ein architekturgeschichtliches Novum“18 gelten kann, denn bislang existierten lediglich Hafensäulen, auf denen Feuer entfacht wurde - wie z. B. im Piräus ${ }^{19}$ oder auf Thasos ${ }^{20}$.

Die vielfach vertretene Meinung, dass das Aussehen wenig interessant war, weil es einige Jahrhunderte später viele dieser Türme gab, ist m. E. nicht zutreffend, da auch die frühen Quellen den Pharos nur selten erwähnen und nie detailliert über ihn berichten, obwohl er allein durch seine Höhe und den Einsatz damaliger moderner Technik zweifellos auffallend gewesen sein muss.

Spätere, arabische Schriften überliefern hingegen teils minutiöse Beschreibungen und Maße, die für die heutige Rekonstruktion grundlegend sind. Sie lassen auf einen dreistufigen Bau, umgeben von einer mit Wehrtürmen besetzten, rechteckigen Terrasse schließen, dessen erstes Geschoss rechteckig, das zweite oktogonal und das dritte zylindrisch gestaltet war $^{21}$ (Abb.1).

Es bleibt die Frage, was den Pharos dennoch, wenn auch spät, zum Weltwunder erhob. Erst mit Gregor von Tours ist im 6. Jh. n. Chr. der Leuchtturm in den Weltwunderlisten belegt, allerdings in Verbindung mit anderen, eher selten benannten Bauten: Es heißt: „Viel anderes Neues gibt es, was die Menschen für bewundernswert halten: ...den Pharos von Alexandria, der auf vier hölzernen Krebsen stehen soll, wenn's denn wahr ist. Man überliefert, dass ein Mensch, der sich über die Schere eines der Krebse ausgestreckt legt, diesen nicht abzudecken vermag ${ }^{622}$.

\footnotetext{
${ }^{15}$ Herodian. 2, 124-125.

${ }^{16}$ Nach der Eroberung Ägyptens 30 v. Chr. Vgl. Aly (1990) 82.

${ }^{17}$ Grimm (1998) 43. Die Zisterne wurde von einer Leitung aus der Stadt gespeist: nach einem arabischen Bericht des Dimaschei: S. dazu Thiersch (1909) 62.

${ }^{18}$ Grimm (1998) 43.

${ }^{19}$ Aus dem 5. Jh. v. Chr.: Thiersch (1909) 19; Empereur (1998) 33; Weltwunder (2003) 175.

${ }^{20}$ Aus dem 6. Jh. v. Chr. mit erhaltener Inschrift. Die Anlage maß 3,5 m im Durchmesser, 2,5 $\mathrm{m}$ in der Höhe. Vgl. Empereur (1998) 33; allg. auch: Sintès (2003) 123-138.

${ }_{21}$ Eine untere Terrasse umgab den Turm, an den Ecken waren Wachtürme angebracht. Der quadratische Grundriss des Baus ma $30 \times 30 \mathrm{~m}$, die untere Etage war $71 \mathrm{~m}$ hoch und verjüngte sich leicht, darauf befand sich ein 8-eckiger, $34 \mathrm{~m}$ hoher Turm, auf dessen Plattform sich wiederum ein zylindrischer Aufbau erhob.

Eine gute Darstellung des Pharos befindet sich auf einem Mosaik in St. Marcus in Venedig aus dem 13. Jh. Diese gibt den Turm ohne Bekrönung wieder (Eckschmitt (1984) Taf. 46a; Empereur (1998a) 85). Auch Karten wie die Tabula Peutingeriana bilden den Turm ab (Thiersch (1909) 25). Kupferstiche von Maarten van Heemskerck (München Graphische Sammlung, Inv.-Nr. 119073) oder von Antonio Tempesta aus dem 16. Jh. (Stendal, Winckelmann-Museum, Inv.-Nr. WG-B-122) geben einen eher phantastischen Eindruck des Bauwerkes.

${ }^{22}$ Vgl. Brodersen (1992) 123.
} 
In den Listen des Mittelalters und denen der Renaissance wird der Leuchtturm häufig erwähnt und schafft es mitunter gar, die stets als beachtlich gelobten Pyramiden $\mathrm{zu}$ verdrängen ${ }^{23}$. Doch ist auch hier immer wieder die Rede von bronzenen, marmornen, selbst gläsernen Krebsen in den Substruktionen, die den Turm tragen. Dies lässt vermuten, dass der Pharos - und zu bemerken sei hier, dass seine Berühmtheit mit stetigem Verfall zunahm - nicht als technisches Wunder galt, sondern aufgrund der obskuren Krebse ${ }^{24}$ erwähnenswert war. Diese tauchen vermehrt in arabischen Quellen ${ }^{25}$ auf, doch ist die Überlieferung ebenso im griechischen Osten bekannt $^{26}$. Eine mögliche Erklärung für diese Merkwürdigkeit könnten die von Augustus 13/12 v. Chr. aufgestellten Obelisken ${ }^{27}$ vor dem Caesareum in Alexandria bieten, von denen einer auf heute noch erhaltenen bronzenen Krebsscheren errichtet war $^{28}$. Der nicht weit vom Pharos entfernte Obelisk mag deshalb Anlass zur Verwechslung geboten haben. Ober aber man war versucht, das Bauwerk, das nun schon deutliche Spuren des Verfalls zeigte, mittels der Krebse interessanter und vor allem ungewöhnlicher darzustellen. Zudem existieren auffällige inhaltliche Parallelen in den Weltwunderlisten: So heißt es bei Pseudo-Beda ${ }^{29}$, dass das Theater von Herakleia aus einem Monolithen gearbeitet war und über sieben steinernen Krebsen in der Schwebe gehalten wird.

Doch scheinen letztlich sämtliche Überlieferungen dieser Art in das Reich der Legenden zu gehören ${ }^{30}$. Bezeichnenderweise datieren die meisten Quellen, die den Pharos mit den Krebsen in Verbindung bringen, in eine Zeit, als der Leuchtturm seiner Hauptaufgabe, den Schiffen den Weg zu weisen, bereits beraubt war, denn seit dem 9. Jh. wurde der Turm von einer Moschee bekrönt, was die Möglichkeit der Befeuerung ausschließt. So zeigt ebenfalls eine Weltwunderliste des 13. Jhs., dass der eigentliche Zweck des Turmes nicht mehr allgegenwärtig war. Es heißt hier: „Der Pharos ist ein hochgelegener Ort, auf den hinaufgestiegen man eine weite Entfernung überblicken kann, wie ich meine. ‘31

\footnotetext{
${ }^{23}$ Aus dem Jahr 1435 stammt die letzte Äußerung zum Turm von Cyriacus von Ancona, der von der immensen Höhe des Pharos schreibt. S. dazu: Hairy (2006) 44.

${ }^{24}$ In einem Bericht des Beda heißt es, der Pharos sei auf vier gläsernen Krebsen 20 Fuß unter dem Meeresspiegel aufgebaut. Etwa zeitgleich (in das 10. Jh.) datiert ein weiterer Vermerk des Ibn Rusta, der vier gläserne Krebse erwähnt. Der Autor Masudi schreibt, dass der Erbauer das Fundament in Form eines gläsernen Krebses auf Meeresbodenniveau habe errichten lassen. Danach schweigen die arabischen Quellen bis zum endgültigen Verschwinden des Bauwerkes. In Wiederholung älterer Autoren kommen seitdem die Krebse wieder zur Erwähnung wie z. B. bei Sujuti, der Gewölbe aus Glas auf dem Rücken von Krebsen im Unterbau beschreibt. Ibn el-Faqih berichtet von einem Krebs aus Glas und von zwei Stützen in Tierform aus Bronze. (Quellen in Übersetzung bei Thiersch (1909) 38-52).

${ }^{25}$ Diese konzentrieren sich vorwiegend auf den Zeitraum von 900-960 n. Chr.

${ }^{26}$ So nennt Kosmas von Jerusalem (677-752 n. Chr.) den Pharos, der auf vier gläsernen Krebsen stehen soll. Für die Glasverwendung am Leuchtturm spricht sich auch Epiphanios von Jerusalem um $800 \mathrm{n}$. Chr. aus. S. auch Brodersen (1993) 209.

${ }^{27}$ Hayward (1978); D'Alton (1993) Nr. 4, 1-72.

${ }^{28}$ Beide Obelisken wurden nach London bzw. New York verbracht. Vogel (2000) 94-101.

${ }^{29}$ Pseudo Beda, Venerabilis 672-735.

${ }^{30}$ Thiersch (1909) 68 geht davon aus, dass die Krebse tatsächlich existierten. Auch Brodersen (1993) 210 sieht die Krebse als passenden Bauschmuck in den Substruktionen.

Die Krebse sind weder in Verbindung mit der Stadt oder dem Turm selbst zu bringen. So ließen die ptolemäischen Könige als Errichter des Pharos keinerlei Bezug zu den Tieren erkennen. Die Münzen der Ptolemäer, üblicherweise oftmals mit Motiven heiliger Tiere (Athen - Eule, Agrigent - Krabbe) versehen, bilden nie Krebs oder Krabbe ab. Oft hingegen sind Adler und Blitzbündel oder Füllhörner dargestellt. Einzig eine Alexandria-Prägung des Antoninus Pius stellt eine Krabbe gemeinsam mit der Büste der Göttin Selene dar, die jedoch als singulär gelten muss.

${ }_{31}$ Es handelt sich um einen anonymen Autor einer Weltwunderliste. Abgedruckt bei Brodersen (1992) 133.
} 
Eine Renaissance erlebte der Leuchtturm erst zu Beginn des letzten Jahrhunderts, als ihn Hermann Thiersch mit einer umfassenden Monographie wieder ins Bewusstsein brachte ${ }^{32}$. Die Arbeit ist auch heute noch für viele Aspekte hilfreich. So machte Thiersch auf die Vorbildwirkung des Pharos für die Minarettbaukunst ${ }^{33}$ aufmerksam, doch sind seine Ausführungen teilweise zu relativieren. m. E. ist die Aussage, dass der Pharos eine spezielle Nachwirkung sowohl bei gotischen Türmen als auch mittelalterlichen Glockentürmen zeitigte ${ }^{34}$, abzulehnen. Doch ist die Gestalt früher ägyptischer und spanischer Minarette durchaus mit dem Pharos vergleichbar. Diese wiesen massive rechteckige Unterbauten auf und waren meist dreietagig. Als Beispiele können hier das Minarett der Hauptmoschee in Kairouan aus dem 7. Jh. (Abb. 2) oder auch das Minarett der Ibn-Tulun-Moschee in Kairo, im 9. Jh. errichtet, genannt werden.

Weiterhin wurde die arabische Bezeichnung „el Manarah“ für einen Ort, an dem Feuer brennt, auch für nordafrikanische Minarette verwendet ${ }^{35}$, was ebenfalls eine deutliche Parallele aufzeigt. Darüber hinaus dürfte die Umfunktionierung des Leuchtturmes zu einer Moschee und der damit einhergehende architektonische Zusammenhang auch Anlass geboten haben, den Pharos in Verbindung mit den Minaretten zu bringen.

Während sich die Forschung bei der Rekonstruktion der Gestalt des Turmes weitestgehend einig ist, bestehen zahlreiche Hypothesen zu Fragen, die den Stifter, die einst bekrönende Statue und das Baumaterial des Pharos betreffen, denn erhalten blieben nur dessen Fundamente, auf denen nach seiner endgültigen Zerstörung im 14. Jh. infolge mehrerer Erdbeben der Sultan Kait Bay ein Hafenkastell errichten lie $\beta^{36}$ (Abb. 3).

Verschiedene Quellen überliefern die Weihinschrift des Turmes, die besagt, dass Sostratos von Knidos, Sohn des Dexiphanes, den Turm den Rettenden Göttern ${ }^{37}$ zum Wohl derer, die zur See fahren, errichtete ${ }^{38}$, wodurch die Frage aufgeworfen wird, ob es sich um den Erbauer oder den Stifter selbst handelt, denn die namentliche Erwähnung eines Architekten wäre im Gegensatz zu der des Finanziers nicht nur unüblich, sondern auch singulär. Die Rolle des Sostratos war vielschichtig: In einer Textpassage bei Sextus Empiricus ${ }^{39}$ wird Sostratos von Ptolemaios I. mit Anfragen zu Antigonos geschickt, woraus $\mathrm{zu}$ schlussfolgern ist, dass er offensichtlich diplomatische Dienste für den Herrscher übernahm. Auch Strabon ${ }^{40}$ erwähnt ihn als einen Freund des Königshauses; er kann somit kein einfacher Architekt gewesen sein, wirkte aber dennoch als ,Ingenieur' auch an anderen Bauten ${ }^{41}$. Es ist indes

\footnotetext{
32 Thiersch (1909); Thiersch (1915) 223-231. Ebenfalls zum Leuchtturm: Veitmeyer (1900) 9-17.

${ }^{33}$ Thiersch (1909) 97; so ebenfalls Butler (1902) 398.

${ }^{34}$ Thiersch (1909) 201.

${ }^{35}$ Thiersch (1909) 172; Eckschmitt (1984) 195 f.

${ }^{36}$ Der Pharos wurde auch durch Caesars Truppen besetzt (Caes. Civ. 3, 112).

37 Als „rettende Gottheiten“ werden entweder die Dioskuren oder Ptolemaios I. und dessen Gattin Berenike vorgeschlagen.

${ }^{38}$ Lukian. 62.

${ }^{39}$ Sextus Empiricus, adv. gramm. 276.

${ }^{40}$ Strab. 17, 6, 791; Plin. nat. 36, 83.

${ }^{41}$ So schreibt Lukian. 2, dass Sostratos seinen König Ptolemaios die Einnahme von Memphis durch die Umleitung des Nil bzw. die Schaffung von Kanälen ermöglichte. Sostratos gilt weiterhin als Erbauer des Terrassenheiligtums der Aphrodite auf Knidos (pensilis ambulatio) nach dem Vorbild der Hängenden Gärten der Semiramis: Plin. nat. 36, 12, 18. Auch in Delphi schuf er verschiedene Gebäude: EDelph III 1, Nr. 298, 299, die sich jedoch nicht erhalten haben.
} 
anzumerken, dass der Betrag von 800 Talenten schwerlich von einem Privatmann aufgebracht worden sein $\mathrm{kann}^{42}$, weswegen die Kosten wohl die ptolemäische Königsfamilie übernahm ${ }^{43}$, wie es auch Plinius mit den Worten ,ein vom König erbauter Turm“ bestätigt. Somit ist die ungewöhnliche Weihinschrift für den Architekten wohl als Initiative des Ptolemaios aus Dank für erwiesene Leistungen und Dienste zu werten.

Es wurde gar verschiedentlich angenommen, dass Sostratos nur die bekrönende Statue des Pharos stiftete ${ }^{44}$, eine Vermutung, die aufgrund der Weihinschrift allerdings wenig plausibel scheint. Um welche Gottheit es sich hierbei handelt, ist in der Forschung umstritten.

Anhaltspunkte zur Lösung dieser Frage, vor allem aber Kontroversen rufen verschiedenste Darstellungen hervor, die sich kontinuierlich auf alexandrinischen Münzen während der Herrschaft der Kaiser Domitian bis Commodus verfolgen lassen: Frühe Münzen bilden nur den Turm ab. Es folgen Darstellungen mit der Göttin Isis als Beschützerin der Seefahrer und dem Pharos. Die späten Münzen zeigen den Leuchtturm in Verbindung mit einem nahenden Schiff (Abb. 4-6). Das Gesamtbild ist zwar recht unzuverlässig, denn die Gestalt des Bauwerkes variiert stark ${ }^{45}$, aber muschelhornblasende Tritonen oberhalb des ersten Stockwerkes und eine bekrönende Figur treten als konstante Elemente auf.

Weiterhin liefern Gefäße, Gemmen sowie Mosaike verschiedene Illustrationen des Pharos. So zeigt eine Glasvase des 2. Jhs. v. Chr. aus Begram ${ }^{46}$ als bekrönende Figur den Meeresgott Poseidon mit einem Ruder; ein spätantikes Mosaik aus Libyen ${ }^{47}$ stellt hingegen eine mit Strahlenkranz versehene Helios-Statue dar, und eine Gemme ${ }^{48}$ zeigt schließlich Isis und Poseidon neben dem Turm, wodurch als bekrönende Statue Zeus zu vermuten ist. Aus den variantenreichen Abbildungen resultierend, entwickelte sich ein breites Meinungsspektrum zur Benennung der Pharos-Statue ${ }^{49}$.

Vertreten werden somit neben Poseidon ${ }^{50}$, Zeus ${ }^{51}$ und Helios ${ }^{52}$ auch alle drei Götter in zeitlicher Folge ${ }^{53}$, oder es wird die vergöttlichte Figur des Ptolemaios I. Soter favorisiert ${ }^{54}$. Dass sich in der christlichen Antike, spätestens ab Theodosius, keine Skulptur einer heidnischen Gottheit auf dem Turm befand, scheint fraglos ${ }^{55}$, doch welcher Gott bekrönte den Pharos in den Jahrhunderten davor? Aufschlussreich

\footnotetext{
${ }^{42}$ Nach Pfrommer (1999) 11 wurde der Turm von Ptolemaios finanziert, aber von einem Privatmann dediziert.

${ }^{43}$ So auch bei Plin. nat. 36,83 .

${ }^{44}$ Empereur (1998) 51.

${ }^{45}$ Der Turm wird sowohl zwei- als auch vieretagig wiedergegeben.

${ }^{46}$ S. dazu: Giorgetti (1956) 247 Taf. 2, 1; Bedon (1988) 60; Wetzel (2005) 503-510.

${ }^{47}$ Giorgetti (1956) 256 Taf. 4, 2; Goodchild (1961) 219 Taf. 39a; Bedon (1988) 62.

${ }^{48}$ Empereur (1998) 50.

${ }^{49}$ Allg. zu Fragen der bekrönenden Statue: Giorgetti (1956) 245-261; Quet (1984) 807-813.

${ }^{50}$ Thiersch (1909) 13.

${ }^{51}$ So Pfrommer (1999) 12; Grimm (1998) 43; Pfrommer (2002) 27; Weltwunder (2003) 175.

${ }^{52}$ Goodchild (1961) 217-223.

${ }^{53}$ Empereur (1998) 51. Somit ergäbe sich diachron: 1. Zeus, 2. Poseidon seit 30 v. Chr., 3. Helios bis zur Herrschaft des Theodosius $391 \mathrm{n}$. Chr.

${ }^{54}$ Picard (1952) 74. Diese Variante schließt sich m. E. schon aufgrund der Tatsache aus, dass der Turm zur Regierungszeit des Ptolemaios I. begonnen und während der Herrschaft des Ptolemaios II. vollendet wurde. Somit hätten beide Herrscher mit einer Statue bedacht werden müssen.

${ }^{55}$ Empereur (1998) 51 geht von einer Christusstatue oder der des heiligen Marcus, dem Stadtpatron, aus.
} 
ist hier ein Epigramm des Poseidippos von Pella ${ }^{56}$ aus dem 3. Jh. v. Chr., in dem steht:

„der sich Alexandria nähernde Seemann wäre sicher, da er - oh Proteus - sein Ziel Zeus Soter (Zๆvòs) nicht verfehlen würde. ‘57

m. E. ist dies ein deutliches Indiz für die einstige Statue des rettenden Zeus. Dass Ptolemaios I. selbst den Beinamen Soter trug, weist außerdem eine Parallele zur Zeus-Statue auf. Darüber hinaus ist überliefert, dass Zeus der Stammvater der ptolemäischen Dynastie ist, denn eine Inschrift besagt ,....abstammend väterlicherseits von Herakles, dem Sohn des Zeus und mütterlicherseits von Dionysos, gleichfalls Sohn des Zeus... ${ }^{58 ،}$.

Eine weitere ungelöste Frage ist die nach dem Material des Turmes. Ausgangspunkt dieses Problems bildet ein Vermerk von Strabon, der das Gestein des Pharos als $\lambda \varepsilon v \kappa o \varsigma \lambda i ́ \theta$ os benennt ${ }^{59}$ - was zunächst „,weißer, leuchtender Stein“ heißt, aber auch als die gebräuchliche Bezeichnung für Marmor als dem leuchtendsten Stein gilt. Somit wurde bislang angenommen, dass der Turm aus Marmor bestand, der aber in Alexandria nicht vorkommt, sondern nur verschiedenfarbiger Granit, Alabaster und Kalkstein.

Die seit den 90er Jahren des 20. Jhs. wiederaufgenommenen Unterwasserforschungen im Areal des Pharos unter der Leitung von J.-Y. Empereur erbrachten, dass die großen, sehr qualitätvollen Blöcke des Fort vermutlich einst zum Pharos gehörten, und diese bestehen aus Kalkstein. So ist zu konstatieren, dass

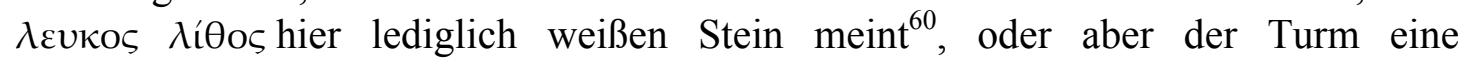
Verkleidungsschicht aus Marmor trug, die nach seiner Zerstörung sekundär für Bauten der Stadt verwendet wurde. Das völlige Fehlen von Marmor im Umkreis des ehemaligen Standortes lässt indes darauf schließen, dass der Bau tatsächlich aus Kalkstein bestand ${ }^{61}$. Auch existierten im 3. Jh. v. Chr. keinerlei Marmorbauten in Alexandria. Man verwendete stattdessen ausschließlich ägyptischen Stein.

Jüngst durchgeführte Tauchkampagnen im Hafenbecken erbrachten weitere Informationen zum Material: So fand sich unter zahlreichen Architektur- und Skulpturenresten ein fragmentierter Türrahmen von fast $13 \mathrm{~m}$ Höhe aus Rosengranit, der als Monumentaleingang dem Pharos zugewiesen wurde und darauf hindeutet, dass Tür- und vermutlich auch Fensterlaibungen aus andersfarbigem Material gestaltet waren $^{62}$. Weiterhin entdeckte man an der Hafeneinfahrt ${ }^{63}$ große Basen, auf denen einst

\footnotetext{
${ }^{56}$ Vgl. dazu: Chamoux (1975) 214-222.

${ }^{57}$ Hom. Od. 4, 354 f.: ,Eine der Inseln liegt im wogenstürmenden Meere Vor des Ägyptos Strome, die Menschen nennen sie Pharos ... Proteus' Tochter, des starken wogenbeherrschenden Greises ...".

58 Fraser (1998) Bd. I 203, 208; Pfrommer (1999) 20. Diese Inschrift gibt der Mönch Kosmas Indikopleustes in seiner „Christlichen Topographie“ wieder.

${ }^{59}$ Strab. 17, 6, 791 .

${ }^{60}$ Die Ansicht von Bernand (1997) 135, dass Strabon irrte, ist nicht zu vertreten.

${ }^{61}$ Bernand (1997) 135 schließt nicht aus, dass man sich für marmorne Gräber und Bauten in Alexandria am Steinbruch des Pharos bediente. Rodziewicz (1984). Für viele Bauten, vorwiegend im Grabkontext, kam Marmor erst in der Spätantike zur Verwendung.

${ }^{62}$ Empereur (1998a) 70; Empereur - Grimal (1997) 698-700.

${ }^{63}$ Aus dem Wasser geborgen wurden Säulen, zwei Naiskoi, Sphingen, neun Figuren aus Rosengranit, davon zwei monumentale (ca. 10,5 m Höhe), vier große (ca. 6,3 m Höhe), eine mittelgroße (ca. 4,2 m Höhe), zwei kleine (ca. 2 m Höhe); deren Stil ist ägyptisch-pharaonisch, mit einzelnen Elementen hellenistischer Manier. Weiterhin fanden sich ein Obelisk von Sethos (1300-1290 v. Chr.) und Papyrussäulen, die von Pfrommer a. O. 15 als Überreste eines Hallenbaus gedeutet werden. S. auch:
} 
sechs Kolossalstatuen von ca. 12 m Höhe standen, die ptolemäische Herrscherpaare möglicherweise die ersten drei Generationen - im altägyptischen Stil darstellten ${ }^{64}$. Drei von ihnen sind fragmentarisch erhalten ${ }^{65}$. Der Rückgriff auf die altägyptische Kunst diente gewiss der Verdeutlichung der Macht der Ptolemäer als makedonische Herrscher und - wohl vor allem - als ägyptische Pharaonen, wodurch zudem die herrscherliche Kontinuität und intendierte Nachfolge deutlich doku-mentiert wurde. Dieser bewusste Rückgriff wird ebenfalls durch die zahlreich aufgefundenen traditionell altägyptischen Statuen und Originalbauteile unterstrichen ${ }^{66}$.

Der Pharos selbst erfüllte somit nicht nur eine profane Aufgabe, sondern war, am Eingang zur Stadt, ein prädestiniertes Areal für den Herrscherkult. Auch war dieser Ort seit jeher ein sakrales Gebiet, in dem beispielsweise die Götter Proteus und Isis verehrt wurden ${ }^{67}$.

Wenn auch der Pharos in den Weltwunderlisten erst zu spätem Ruhm gelangte, so ist ihm zumindest eine architektonische Vorbildwirkung zuzuweisen. Die deutlichsten baulichen Reminiszenzen scheinen bei einem späthellenistischen Grabmal aus Taposiris Magna ${ }^{68} \mathrm{zu}$ bestehen, das sich ca. $50 \mathrm{~km}$ von Alexandria entfernt befindet (Abb.7).

Auch wurde das Motiv des Turmes für antike Souvenirs in die alexandrinische Terrakottaproduktion übernommen ${ }^{69}$. Das Bestreben zur Nachahmung ist evident und reicht über den persönlichen Gestaltungsgeschmack einzelner Grabinhaber und Nachbildungen touristischer Attraktionen hinaus, denn der Pharos beeinflusste die später entstehenden Leuchttürme in der antiken Welt. Als eines der frühesten bekannten Beispiele kann zunächst der Leuchtturm von Messina gelten, der auf zahlreichen Münzen des Pompeius abgebildet ist (Abb. 8). Weiterhin sind der Turm in Forum Julii ${ }^{70}$ (Fréjus), unter Caesar erbaut, der Leuchtturm in Gesoriacum (bei Calais), mit dessen Bau während der Regierungszeit des Caligula begonnen wurde ${ }^{71}$, ein anderer in Dubris ${ }^{72}$ (Dover), den Kaiser Claudius errichten ließ und schließlich der

Empereur (1998) 85-95, 115-119; Yoyotte (1998) 199-212; Empereur (1998a) 65-81; Hairy (2006) 4649.

${ }^{64}$ Es handelte sich vermutlich um die Paare Ptolemaios I. und Berenike I., Ptolemaios II. und Arsinoe II., Ptolemaios III. und Berenike II., die im Zeitraum von 300-222 v. Chr. ihre Herrschaft ausübten. Schon vor seinem Regierungsantritt lässt sich Ptolemaios in seiner Funktion als Satrap wie ein ägyptischer Pharao darstellen: So auf der sog. Satrapenstele (Hildesheim, Pelizaeus-Museum Inv. 1883). Dazu auch: Hölbl (1994) 75 f. Abb. 5; Empereur (1998b) 180-182. Einen deutlichen Bezug zu ägyptischen Bräuchen beweist auch der Übergang von der bislang geübten Brandbestattung zur Mumifizierung, die durch die ersten drei ptolemäischen Könige eingeleitet wurde: Grimm (1997) 244.

${ }^{65} 1995$ wurden drei Kolossaalköpfe der Ptolemäer aus Assuangranit geborgen. Eine Königin war an die Göttin Isis angeglichen. Vgl. Empereur - Grimal (1997) 698-700.

${ }^{66}$ S. Fn. 63.

${ }^{67}$ Die Insel war für den Kult des Proteus berühmt: Pfrommer (1999) 11. Auch Isis Pharia genoss Verehrung in einem Tempel nahe dem Pharos. So fand man eine Kolossalstatue der Göttin im Hafenbereich. S. auch: Hairy (2006) 31 f. Auch antike Autoren wie Ovid berichten vom dortigen IsisKult: Ov. met. 9, 773.

68 Empereur (1998) 42; Pfrommer (1999) 13. Die frühere Forschung tendierte zur Deutung als Leuchtturm oder Landmarke: Thiersch (1909) 26-31; el Fakharani (1974) 257-272.

${ }^{69}$ Grunwald (2004) 68 f. Abb. 6.

${ }^{70}$ Der Turm war ca. $25 \mathrm{~m}$ hoch, mit pyramidalem Abschluss und bekrönender Statue. Vgl. Février (1963); Bedon (1988) 59.

${ }^{71}$ Suet. Cal. 46 betont die Höhe des 12-stöckigen, bis 1644 erhaltenen Baus und sein Signalfeuer. Vgl. Seillier (o. J.) 201-211.

${ }^{72}$ Der Turm war ca. $28 \mathrm{~m}$ hoch und mit einem 3 m hohen, runden Aufbau versehen. Vgl. Bedon (1988) 60. 
bekannte Pharos von Ostia zu erwähnen, der gleichfalls unter Claudius begonnen wurde $^{73}$ (Abb. 9). Die meisten der Bauten waren nach Berichten, Münzdarstellungen und anderen Abbildungen drei- bis vieretagig und von unterschiedlichem Grundriss ${ }^{74}$. Von Statuenbekrönungen und Signalfeuern ist auch bei einigen dieser Türme in der antiken Literatur die Rede. Insgesamt sind 17 Leuchttürme überliefert, für weitere 10 ist deren einstige Existenz hypothetisch.

Der alexandrinische Pharos war und blieb der höchste Leuchtturm, kein anderer war auch nur halb so hoch. Die einzige Anlage, die noch heute einen authentischen Eindruck liefert, ist der Turm in Brigantium (La Coruña). Dank zahlreicher Restaurierungen ist der bereits 100 n. Chr. von Servius Lupus erbaute Leuchtturm noch in Funktion ${ }^{75}$ (Abb. 10).

Es lassen sich letztlich nicht im einzelnen Bezüge zum alexandrinischen Pharos herstellen und Vergleiche treffen, doch hat er zweifellos den entscheidenden Anteil dazu beigetragen, die Hafenleuchten durch eine neue Gattung, die der Leuchttürme zu ersetzen. Wie deutlich aber das Vorbild kopiert wurde, muss unbeantwortet bleiben, doch wird auch von Flavius Josephus ${ }^{76}$ und Herodian ${ }^{77}$ berichtet, dass spätere Türme sich in ihrer Gestalt an den Pharos anlehnten und ihm insofern trotz der erst späten Würdigung als Weltwunder ein deutlicherer Nachklang als vielen anderen stets gerühmten Bauwerken beschieden war.

\footnotetext{
${ }^{73}$ Meiggs (1998) 121: Der vieretagige Turm wurde im Jahr 54 n. Chr. eingeweiht. Vgl. Stuhlfauth (1938) 139-163; Bedon (1988) 58; Keay - Millett - Paroli (2005).

${ }^{74}$ Weitere Bauten existierten beispielsweise in Ravenna, Smyrna, Patara, Apameia, Leptis Magna. Eine ausführliche Zusammenstellung der Bauten bietet Reddé (1979) 845-872; Bedon (1988) 56-60.

${ }^{75}$ Der Turm ist $34 \mathrm{~m}$ hoch und besitzt einen quadratischen Grundriss. Vgl. Hutter (1973); Hauschild (1976) 238-257; Hutter (1978) 33-48.

${ }^{76}$ Ios. bel. Iud. 5, 166-169.

${ }^{77}$ Herodian. 4, 2.
} 


\section{Bibliographie}

Aly (1990)

Archaeology (o. J.)

Bedon (1988)

Bernand (1997)

Brodersen (1992)

Brodersen (1993)

Butler (1902)

Chamoux (1975)

Clayton - Price (1991)

D'Alton (1993)

Eckschmitt (1984)

el Fakharani (1974)

Empereur - Grimal (1997)

Empereur (1998)

Empereur (1998a)

Empereur (1998b)

Février (1963)

Fraser (1998)

Giorgetti (1956)

Goddio u. a. (1998)

Goodchild (1961)
RE IV A 1 (Nachdr. 1990) s. v. Strabon 82 (Aly). Archaeology in Confrontation. Aspects of Roman Military Presence in the Northwest, Studies in Honour of H. Thoen (o. J.) 201-211.

R. Bedon, Les phares antiques, Archeologia, Paris 231, 1988, 54-66.

A. Bernand, Le „marbre“ du Phare d'Alexandrie, ZPE 118, 1997, 131-138.

K. Brodersen, Reiseführer $\mathrm{zu}$ den Sieben Weltwundern (1992).

K. Brodersen, Ein Weltwunder auf ,gläsernen Füßen", Der Pharos von Alexandria in neuem Licht, AW 24, 1993, 207-211.

A. J. Butler, The Arab Conquest of Egypt (1902).

F. Chamoux, L'Epigramme de Poseidippos sur le Phare d'Alexandrie, in: Le Monde grec, Hommages à Claire Préaux (1975) 214-222.

P. Clayton - M. Price, The Seven Wonders of the Ancient World (1991).

M. D'Alton, The New York Obelisk, or how Cleopatra's Needle came to New York and what happened when it got here, BMetrMus 50, 1993, Nr. 4, 1-72.

W. Eckschmitt, Die sieben Weltwunder (1984).

F. el Fakharani, The 'Lighthouse' of Abusir in Egypt, HarvStClPhil 78, 1974, 257-272.

J.-Y. Empereur - N. Grimal, Les fouilles sousmarines de Phare d'Alexandrie, CRAI 1997, 698-700.

J.-Y. Empereur, Le Phare (1998).

J.-Y. Empereur, Alexandria Rediscovered (1998).

J.-Y. Empereur, Recherches récentes a Alexandrie, RA 1998, 180-183.

P. A. Février, Forum Julii (Fréjus) (1963).

P. M. Fraser, Ptolemaic Alexandria (Neudr. 1998) Bd. I.

M. Giorgetti, Il Faro di Alessandria fra simbologia e realtà: dall'Epigramma di Posidippo ai mosaici di Gasr Elbia, Annales du Service des Antiquités de 1'Ègypte 54, 1956, 244-261.

F. Goddio u. a., Alexandria, The Submerges Royal Quarters (1998) 199-212.

R. G. Goodchild, Helios on the Pharos, The Antiquaries Journal 41, 1961, 217-223. 
Grimm (1997)

Grunwald (2004)

Hairy (2006)

Hauschild (1976)

Hayward (1978)

Hölbl (1994)

Hutter (1973)

Hutter (1978)

Keay - Millett - Paroli (2005)

Meiggs (1998)

Oehler (1920)

Pfrommer (1999)

Pfrommer (2002)

Picard (1952)

Quet (1984)

Reddé (1979)

Rodziewicz (1984)

Schoene (1866)

Seillier (o. J.)

Sintès (2003)

Stuhlfauth (1938)
G. Grimm, Verbrannte Pharaonen, Die Feuerbestattung Ptolemaios' IV. Philopator und ein gescheiterter Staatsstreich in Alexandria, AW 3, 1997, 233-249.

L. Grunwald, Alexandrinisches Leben in Ton gebrannt, AW 4, 2004, 65-72.

I. Hairy, Le phare d'Alexandrie, concentré de géométr, Archéologie, La recherche: revue mensuelle Société d'Éditions Scientifiques Paris 37, 2006, 44-51.

T. Hauschild, Der römische Leuchtturm von La Coruña (Torre de Hercules). Probleme seiner Rekonstruktion, MM 17, 1976, 238-257.

R. A. Hayward, Cleopatra's Needles (1978).

G. Hölbl, Geschichte des Ptolemäerreiches. Politik, Ideologie und religiöse Kultur von Alexander dem Großen bis zur römischen Eroberung (1994).

S. Hutter, Der römische Leuchtturm von La Coruña (1973).

S. Hutter, Der antike römische Leuchtturm von La Coruña. Der Turm des Herkules, AW 9, 1978, 33-48.

S. Keay - M. Millett - L. Paroli u. a., Portus. An Archaeological Survey of the Port of Imperial Rome (2005).

R. Meiggs, Roman Ostia (1998).

A. Oehler, Der Kranz des Meleagros von Gadara (1920).

M. Pfrommer, Alexandria. Im Schatten der Pyramiden, Sonderheft AW (1999).

M. Pfrommer, Königinnen vom Nil (2002).

C. Picard, Sur quelques répresentations nouvelles $\mathrm{du}$ Phare d'Alexandrie et sur l'origine Alexandrine des Paysages portuaires, $\mathrm{BCH}$ 76, 1952, 61-95.

H. Quet, Pharus, MEFRA 96, 1984, 807-813.

M. Reddé, La représentation des Phares à 1'époque romaine, MEFRA 91, 2, 1979, 845-872.

M. Rodziewicz, Les habitations romaines tardives d'Alexandrie (1984).

A. Schoene, Eusebi Chronicorum I (1866).

C. Seillier, Le castrum e le port de Gesoriacum (Boulogne-sur-Mer) base de la classis Britannica (IIe-IIIe siècles), in: Archaeology (o. J.) 201-211. G. Sintès, Thasos, un port depuis l'Antiquité ... malgre tout, $\mathrm{BCH} 127,2003,123-138$.

G. Stuhlfauth, Der Leuchtturm von Ostia, RM 53, 1938, 139-163. 
Thiersch (1909)

Thiersch (1915)

Veitmeyer (1900)

Vogel (2000)

Weltwunder (2003)

Wetzel (2005)

Yoyotte (1998)
H. Thiersch, Pharos: Antike, Islam und Occident. Ein Beitrag zur Architekturgeschichte (1909).

H. Thiersch, Griechische Leuchtfeuer, JdI 30, 1915, 223-231.

L. A. Veitmeyer, Leuchtfeuer und Leuchtapparate (1900) (Neudr. 2005).

C. Vogel (überarbeitete Fassung von L. Habachi), Die unsterblichen Obelisken Ägyptens (2000).

Die Sieben Weltwunder der Antike. Wege der Wiedergewinnung aus sechs Jahrhunderten. Ausstellung im Winckelmann-Museum Stendal (2003).

J. Wetzel, Der Pharosbecher aus Begram. Analyse der Schleiftechnik, AKorrB1 25, 2005, 503-510.

J. Yoyotte, Pharaonica, in: F. Goddio u. a. (1998) 199-212.

\section{Abbildungsnachweis}

Abb. 1 Thiersch (1909) Beil 1.

Abb. 2 Thiersch (1909) 124 Abb. 149.

Abb. 4-6 www.CoinArchives.com Ancient Coins Home (Pharos) Nr. 24, $26,7$.

Abb. 8 www.CoinArchives.com Ancient Coins Home (Pharos) Nr. 17.

Alle übrigen Abb. von Verf. 
Frankfurter elektronische Rundschau zur Altertumskunde 3 (2006)

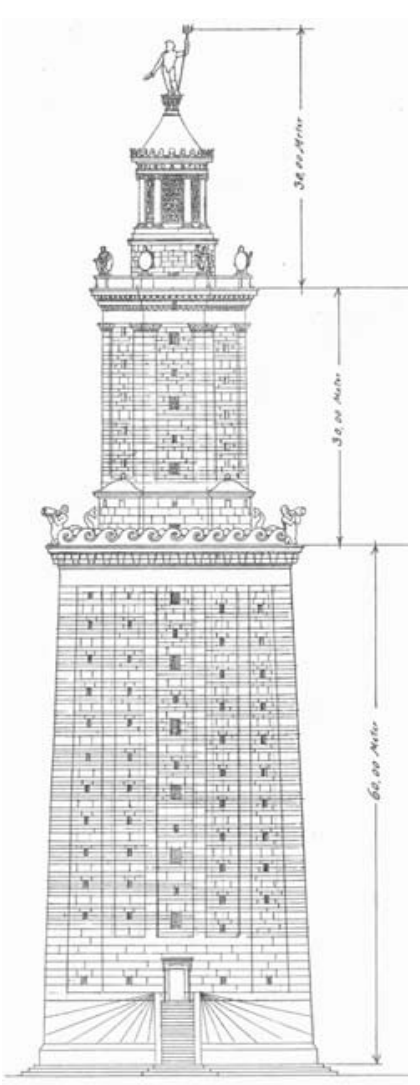

Abb. 1 Rekonstruktion des Pharos nach $\mathrm{H}$. Thiersch

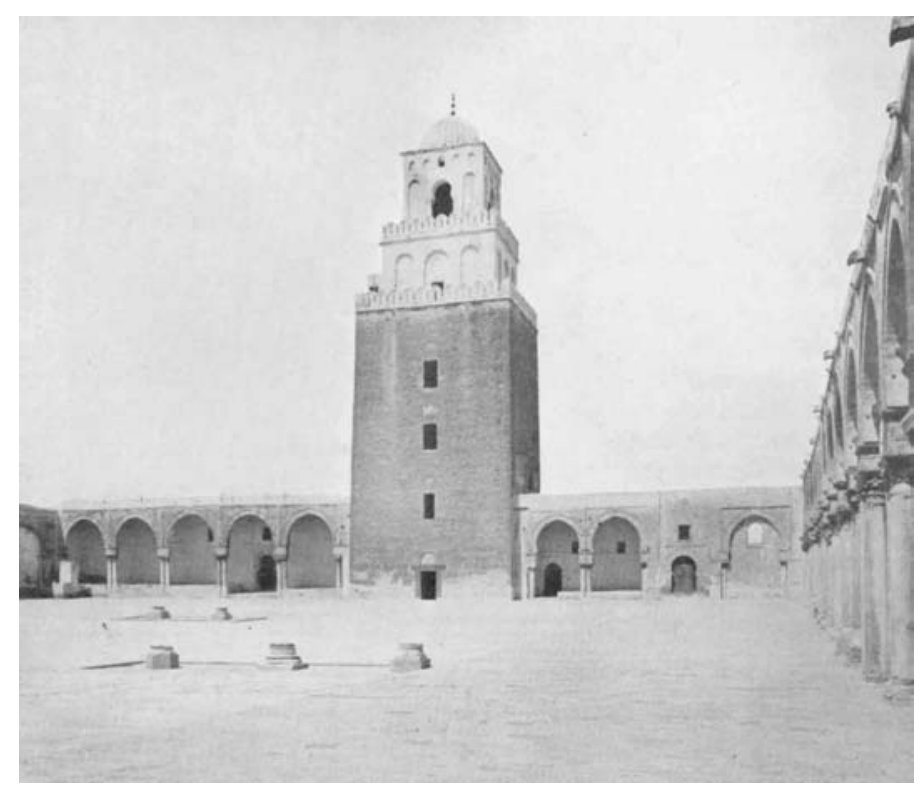

Abb. 2 Minarett der Hauptmoschee in Kairouan

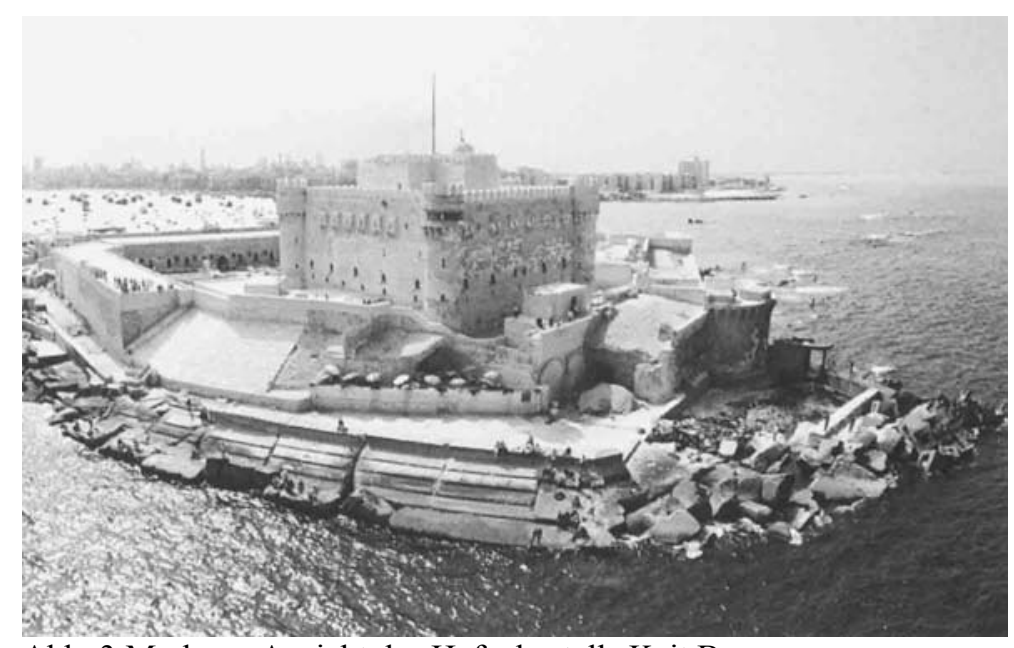

Abb. 3 Moderne Ansicht des Hafenkastells Kait Bay

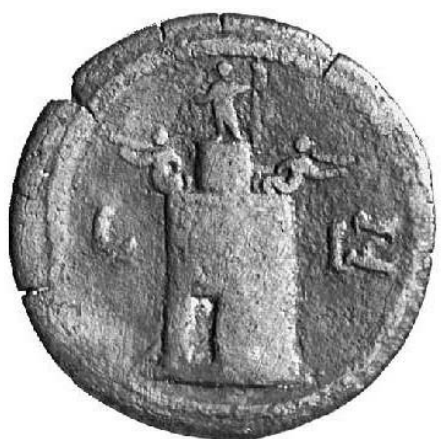

Abb. 4 Pharos auf einer Münze des Hadrian

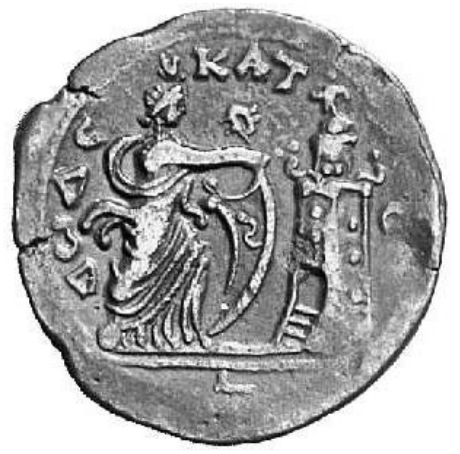

Abb. 5 Pharos auf einer Münze des Antoninus Pius

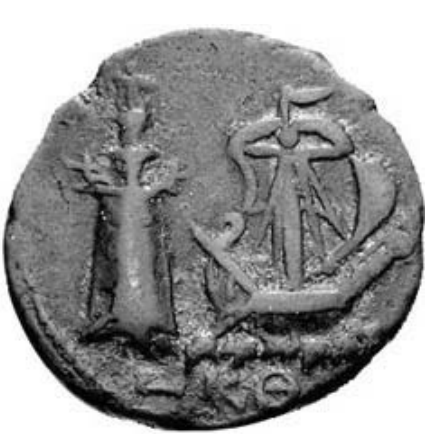

Abb. 6 Pharos auf einer Münze des Commodus 
Frankfurter elektronische Rundschau zur Altertumskunde 3 (2006)

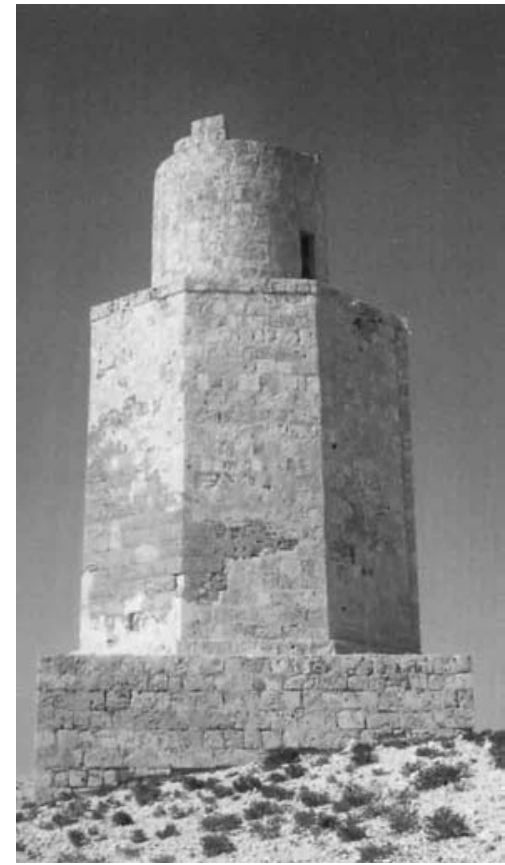

Abb. 7 Das Grabmal von Taposiris Magna

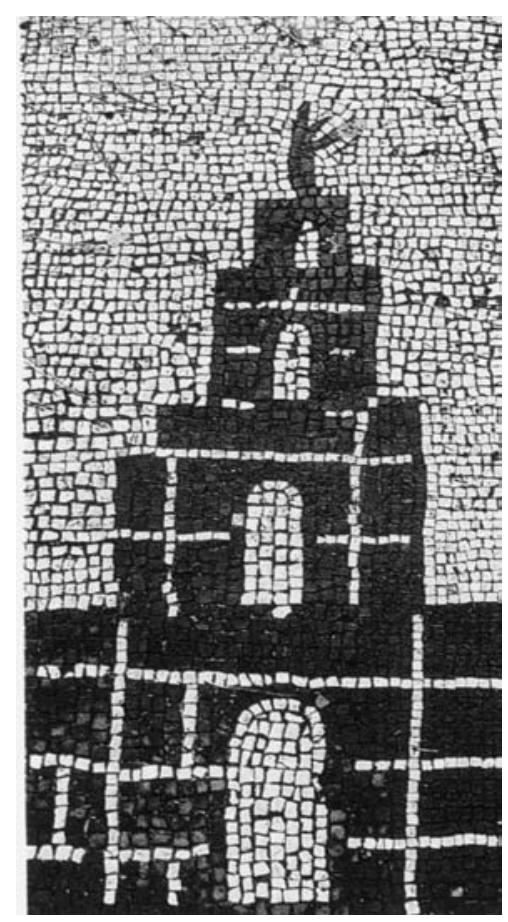

Abb. 9 Mosaik aus Ostia

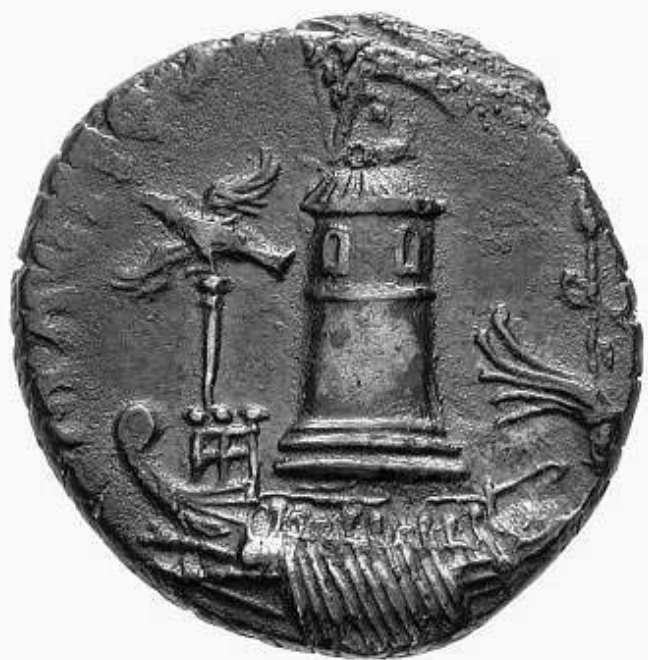

Abb. 8 Der Leuchtturm von Messina auf einer Münze des Pompeius

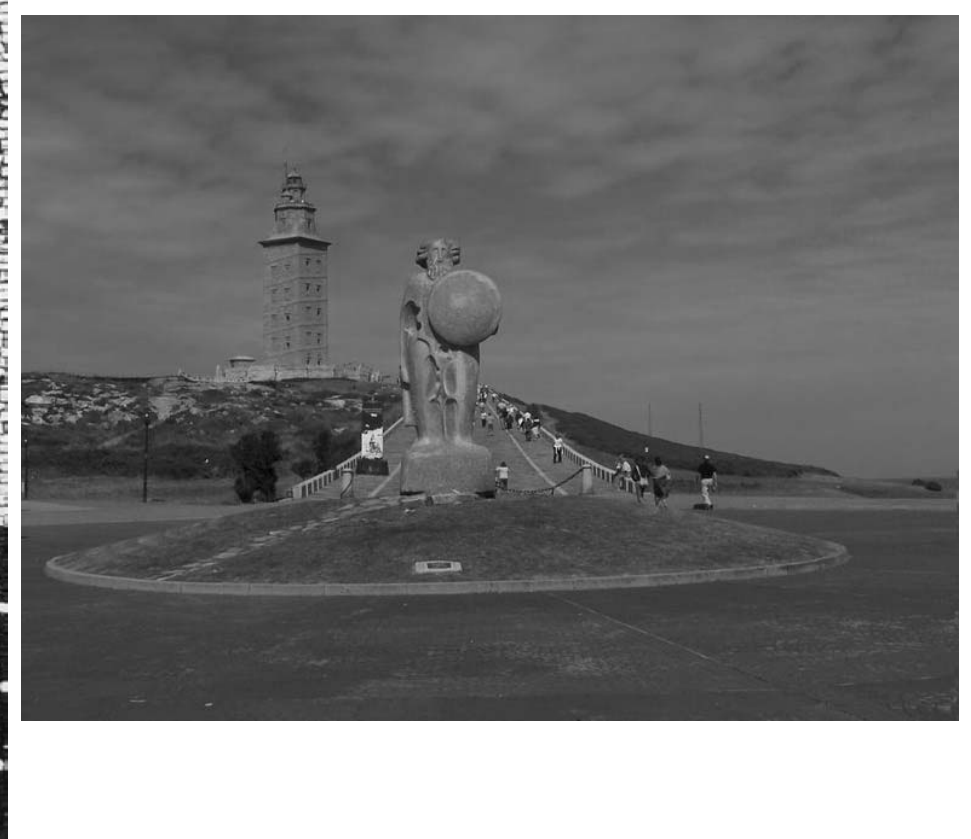

Abb. 10 Der Leuchtturm von Brigantium (La Coruña) 